\title{
The potential role of long noncoding RNAs in primary open-angle glaucoma
}

\author{
Feng Zhang ${ }^{1}$ - Yang Zhao ${ }^{2,3} \cdot$ Mengdan $\mathrm{CaO}^{4} \cdot \mathrm{Xu} \mathrm{Jia}^{5} \cdot$ Zheng Pan $^{4} \cdot$ Dengming Zhou ${ }^{4} \cdot \mathrm{Ke} \mathrm{Liu}^{4} \cdot$ Xuanchu Duan $^{2,3}$
}

Received: 8 February 2021 / Revised: 5 May 2021 / Accepted: 9 June 2021 / Published online: 10 July 2021

(c) The Author(s) 2021

\begin{abstract}
Purpose To identify the potential genes in human trabecular meshwork (TM) related to primary open-angle glaucoma (POAG).

Methods First, long noncoding RNA (LncRNA) and mRNA expression profiles in TM samples from 4 control subjects and 4 POAG patients were accessed by microarray analyses. Then, twenty lncRNAs were validated by real-time quantitative PCR in the same samples from microarray analyses. Finally, eight highly expressed lncRNAs were further tested by realtime quantitative PCR in TM from 8 normal controls and 19 POAG patients. Expression data were normalized and analyzed using the R software. Pathway analyses were performed by Gene Ontology (GO) and Kyoto Encyclopedia of Genes and Genomes (KEGG) analysis.

Results A total of 2179 lncRNAs and 923 mRNAs in the TM of POAG patients were significantly upregulated, and 3111 lncRNAs and 887 mRNAs were significantly downregulated. ENST00000552367, ENST00000582505, ENST00000609130, NR_029395, NR_038379, and ENST00000586949 expression levels were significantly higher in the TM from a different cohort of POAG patient than normal controls.

Conclusion ENST00000552367, ENST00000582505, ENST000006091- 30, NR_029395, NR_038379, and ENST00000586949 may play essential roles in the development of POAG.
\end{abstract}

\section{Key Messages:}

- The pathogenesis of primary open-angle glaucoma is related to long noncoding RNAs.

- We detected long noncoding RNA (LncRNA) and mRNA expression profiles in TM samples from 4 controla subjects and 4 POAG patients were accessed by microarray analyses.

- Eight highly expression lncRNAs were further tested by real-time quantitative PCR in TM from 8 normal controls and 19 POAG patients.

- ENST00000552367, ENST00000582505, ENST000006091 -30, NR_029395,NR_038379, and ENST00000586949 may play essential roles in the development of POAG.

Keywords Long noncoding RNA · Primary open-angle glaucoma · Trabecular meshwork

Xuanchu Duan

duanxchu@126.com

Extended author information available on the last page of the article 


\section{Introduction}

Glaucoma, after cataracts, is the most frequent cause of blindness worldwide [1], affecting more than 60 million people. Also, the number of primary open-angle glaucoma (POAG) patients is estimated to be 45 million around the world [2]. Recognized risk factors for POAG include elevated intraocular pressure (IOP) [3], genetic factors [4], environmental circumstances [5, 6], refractive error [7], and systemic diseases [8,9].

With the discovery and study of noncoding RNAs which contain miRNAs, circular RNAs, and long noncoding RNAs (lncRNAs), the relationship between ncRNAs and diseases has raised concern recently [10]. LncRNAs (ncRNAs > 200 nucleotides in length) have long been regarded as junk RNAs. Recently, however, lncRNAs have been shown to play key roles in a variety of cellular processes through interaction with the main component proteins in gene regulatory systems [11]. Currently, lncRNAs were shown to take part in the biomarker [12], development, and progression of glaucoma [13].

In our study, we performed microarray assays to obtain an overview of the expression profiles of various $1 n c R-$ NAs and mRNAs in the trabecular meshwork of POAG patients and normal subjects. Disease-related lncRNA profiles in the trabecular meshwork of POAG patients have been discovered. We found that ENST00000552367, ENST00000582505, ENST000006091- 30, NR_029395, NR_038379, and ENST00000586949 may play an essential role in the development of POAG.

\section{Materials and methods}

\section{Procurement of trabecular meshwork}

The study conforms to all tenets of the Declaration of Helsinki, and written informed consent was obtained from all subjects. This research was approved by the Ethics Committee of The Second Xiangya Hospital of Central South University (Changsha, China). All donated samples were obtained from The Second Xiangya Hospital.

Trabecular meshwork for test group was obtained from POAG patients who had uncontrolled IOP and accepted trabeculectomy surgery performed by one surgeon (XC. D). The inclusion criteria of POAG were the following: (1) age at POAG diagnosis older than 30 years, (2) glaucomatous optic nerve damage with associated visual field damage, and (3) exclude secondary glaucoma. All control TM tissue was obtained from donor eyes without glaucoma or glaucoma-associated condition.

\section{RNA isolation and qPCR}

TRIzol Reagent (Invitrogen Life Technologies, Carlsbad, CA) was used to extract total RNA from the TM samples. The total RNA quantity and quality were measured by NanoDrop ND-1000. RNA integrity was assessed by standard denaturing agarose gel electrophoresis. Total RNA was also purified with RNeasy MinElute Cleanup Kit (Qiagen, Hilden, Germany) according to the manufacturer's protocol. RNA was reverse transcribed into cDNA with the SuperScript $^{\mathrm{TM}}$ III Reverse Transcriptase (Invitrogen, CA). Then, the cDNA was used for carrying out quantitative RT-PCR which was conducted by SYBR green expression master mix (Applied Biosystems, Inc., Foster City, CA, USA). The forward and reverse primer sequences are listed in Table 2. The $\Delta \Delta \mathrm{CT}$ method $\left(2^{-\Delta \Delta \mathrm{Ct}}\right)$ was applied to calculate the relative differences between the control and POAG groups.

\section{Microarray analysis}

TM RNA samples from 4 control subjects and 4 POAG patients for microarray analyses were extracted and the RNA integrity was tested by standard denaturing agarose gel electrophoresis, as described above. RNA sample labeling and array hybridization were performed according to the Agilent One-Color Microarray-Based Gene Expression Analysis Protocol (Agilent, Santa Clara, CA). Briefly, mRNA was purified from total RNA after removal of rRNA (mRNA-ONLYTM Eukaryotic mRNA Isolation Kit, Epicentre). Then, each sample was amplified and transcribed into fluorescent cRNA along the entire length of the transcripts without $3^{\prime}$ bias utilizing a random priming method (Arraystar Flash RNA Labeling Kit, Arraystar). The labeled cRNAs were purified by RNeasy Mini Kit (Qiagen). The concentration and specific activity of the labeled cRNAs (pmol Cy3/ $\mu$ g cRNA) were measured by NanoDrop ND-1000. One microgram of each labeled cRNA was fragmented by adding $5 \mu 110 \times$ blocking agent and $1 \mu \mathrm{l}$ of $25 \times$ fragmentation buffer, then the mixture was heated at $60{ }^{\circ} \mathrm{C}$ for $30 \mathrm{~min}$; finally, $25 \mu \mathrm{l} 2 \times \mathrm{GE}$ Hybridization buffer was added to dilute the labeled cRNA. Fifty microliters of hybridization solution was dispensed into the gasket slide and assembled to the lncRNA expression microarray slide. The slides were incubated for $17 \mathrm{~h}$ at $65{ }^{\circ} \mathrm{C}$ in an Agilent Hybridization Oven. The hybridized arrays were washed, fixed, and scanned using the Agilent DNA Microarray Scanner (part number G2505C).

Data were extracted through the Agilent Feature Extraction software (Agilent, Santa Clara, CA). All original data have been uploaded to Gene Expression Omnibus public database (https://www.ncbi.nlm.nih.gov/geo; GSE138125). 


\section{GO and KEGG enrichment analysis}

The Gene Ontology (GO) (http://www.geneontology.org) is a major bioinformatic tool to annotate genes and analyze biological process of these genes [14]. Kyoto Encyclopedia of Genes and Genomes (KEGG) (http://www.genome.jp/kegg) is a database resource for understanding high-level functions and biological systems from large-scale molecular datasets [15]. $\mathrm{P}<0.05$ was considered statistically significant.

\section{Statistical analysis}

A train of data processing was performed through the $\mathrm{R}$ software package version 3.6.0 [16]. Numeric variables were compared using t-test. Results were expressed as means \pm standard deviation. All statistical analyses were performed with GraphPad Prism 7 (GraphPad Software, USA). The results were considered significant if $\mathrm{P}<0.05$.

\section{Results}

\section{Demographics and characteristics of POAG cases and controls}

Our study included 23 human TMs from POAG patients and 12 healthy controls who donated their eyes after death from the Second Xiangya hospital, Central South University. The baseline characteristics of the subjects are summarized in Table 1. Subjects in the control and POAG groups were aged $49.83 \pm 10.16$ (mean \pm SD) and $50.43 \pm 9.72$ years, respectively. The male percentage of the control and POAG groups was $58.33 \%$ and $43.48 \%$, respectively.

\section{Microarray expression profiling of IncRNAs and $m R N A s$ in trabecular mesh from individual subjects}

To detect and identify differentially expressed lncRNAs and mRNAs in the trabecular meshwork of POAG and normal patients, tissue samples were collected and quantified by microarray assays (Fig. 1). A total of 2179 lncRNAs and 923 mRNAs were significantly upregulated (fold change $\geq 2$,

Table 1 Clinical characteristics of subjects which trabecular meshwork was used in the study

\begin{tabular}{lll}
\hline Characteristics & $\begin{array}{l}\text { Control } \\
(\mathrm{n}=12)\end{array}$ & $\begin{array}{l}\text { POAG } \\
(\mathrm{n}=23)\end{array}$ \\
\hline $\begin{array}{l}\text { Age, mean } \pm \mathrm{SD} \text {, years } \\
\text { Sex, } \%\end{array}$ & $49.83 \pm 10.16$ & $50.43 \pm 9.72$ \\
$\quad$ Male & 58.33 & \\
Female & 41.67 & 43.48 \\
\hline
\end{tabular}

false discovery rate $\leq 0.05, \mathrm{P} \leq 0.05)$, and $3111 \operatorname{lncRNAs}$ and 887 mRNAs were significantly downregulated (fold change $\geq 2$, false discovery rate $\leq 0.05, \mathrm{P} \leq 0.05$ ), in POAG patients compared with control subjects.

\section{GO and KEGG pathway enrichment analysis}

The Gene Ontology (GO) (http://www.geneontology.org) and Kyoto Encyclopedia of Genes and Genomes (KEGG) pathway enrichment analysis (http://www.genome.jp/kegg) were performed to explore potential functions of differentially expressed genes and correlated pathways. Dot plots (Fig. 2) showed the results of GO enrichment. The upregulated mRNAs (Fig. 2A) include protein glycosylation, macromolecule glycosylation, glycosaminoglycan biosynthetic process, glycoprotein metabolic process, glycoprotein biosynthetic process, establishment of localization, establishment of blood-brain barrier, cellular response to zinc ion, carbohydrate derivative biosynthetic, and aminoglycan biosynthetic process, whereas biological process of downregulated (Fig. 2B) mRNAs includes vesicle targeting, synaptic transmission, glutamatergic, regulation of synaptic transmission, regulation of neurotransmitter levels, protein homo tetramerization, protein homo oligomerization, positive regulation of transmembrane, positive regulation of blinding, negative regulation of cartilage, and golgi vesicle transport.

The KEGG pathway enrichment analyses showed that 10 pathways were significantly enriched in upregulated genes (Fig. 3A), including ECM-receptor interaction, arrhythmogenic right ventricular cardiomyopathy (ARVC), glycosphingolipid biosynthesis-globo and isoglobo series, hypertrophic cardiomyopathy (HCM), malaria, glycosaminoglycan biosynthesisheparan sulfate/heparin, vibrio cholerae infection, glucosaminoglycan biosynthesis-keratan sulfate, PI3K-Akt signaling pathway, and dilated cardiomyopathy. Moreover, ten pathways were enriched in downregulated genes (Fig. 3B), including lysosome, tuberculosis, taurine and hypotaurine metabolism, amino sugar and nucleotide sugar metabolism, alanine, aspartate and glutamate metabolism, fatty acid degradation, arachidonic acid metabolism, fatty acid metabolism, long-term potentiation, and amphetamine addiction.

\section{Real-time quantitative PCR validation}

To confirm the microarray analysis results and identify potential related lncRNA for development of POAG, 20 lncRNAs (Table 2$)$ that had highly significant $P$ values $(P<0.01)$, large fold changes $(>3.0)$, false discovery rate $(<0.025)$, and stated positive correlation with POAG-relevant mRNA are chosen and listed in Table 3. Their expression was assessed by individual RT-qPCR assays using the same samples from the initial microarray analysis (Fig. 4). Consistent with the microarray results, ENST00000422366, ENST00000430429, 
A

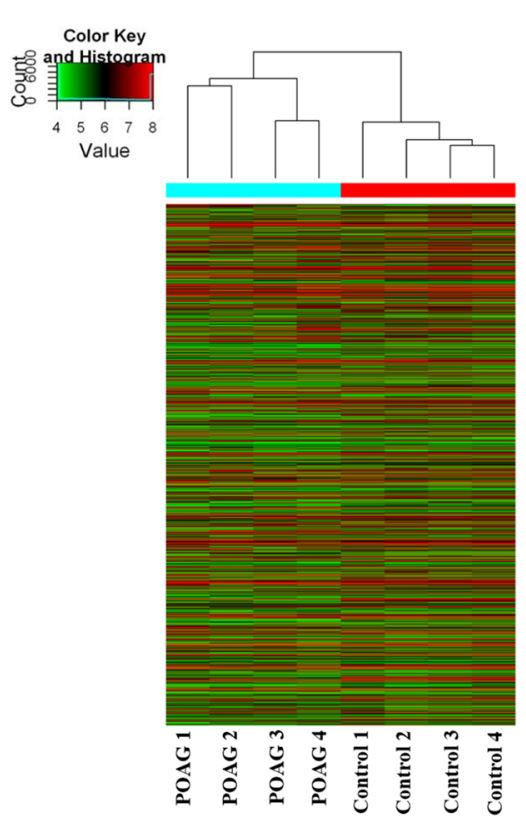

C

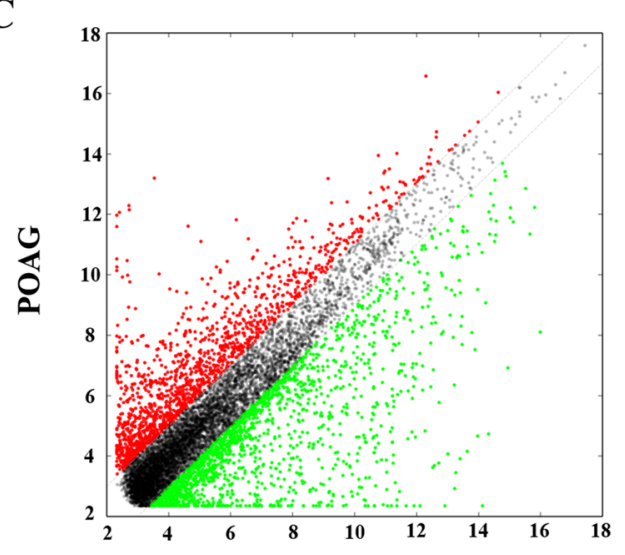

Control

$\mathbf{E}$

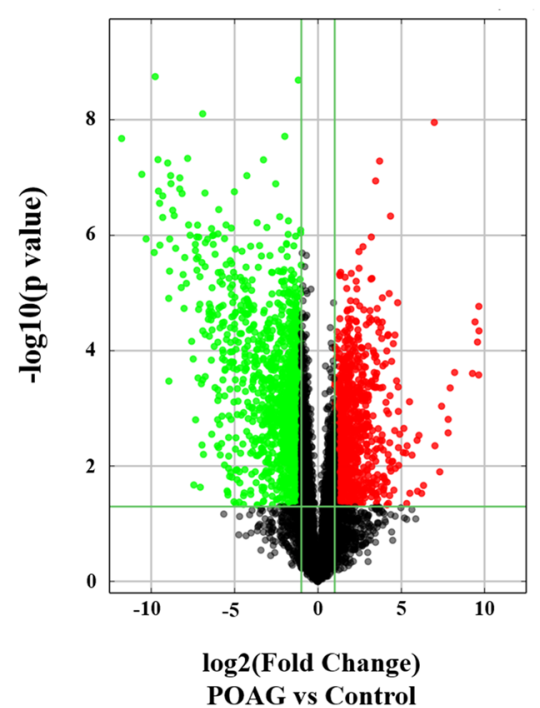

B

mRNA

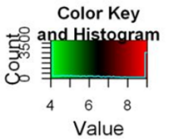
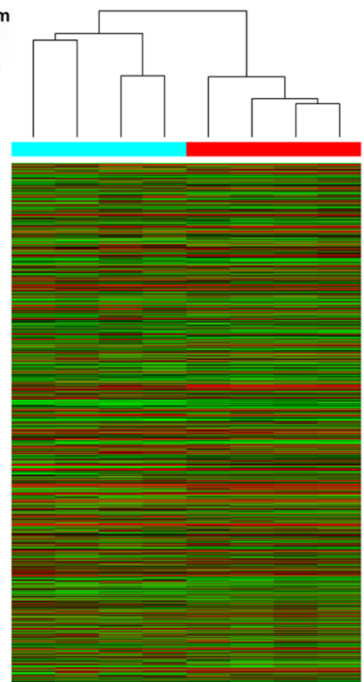

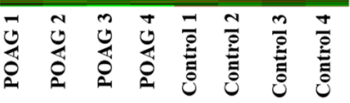

D

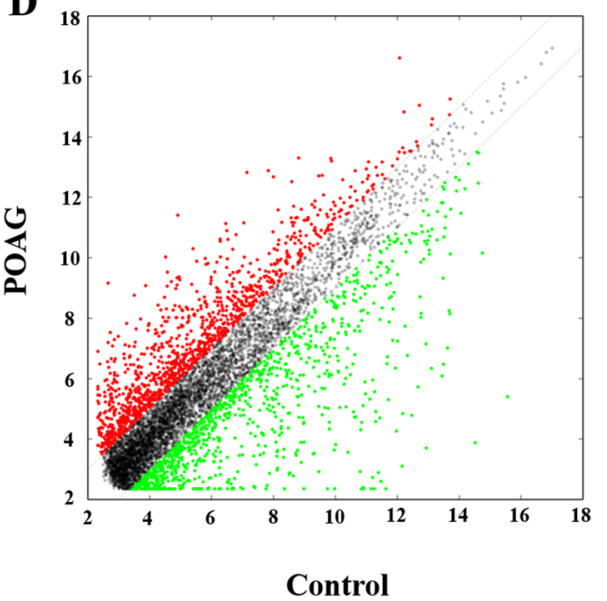

F

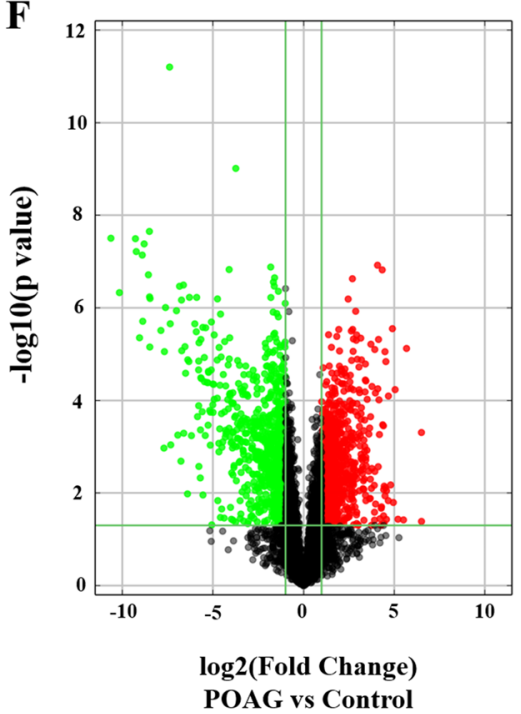


4 Fig. 1 Microarray expression profiling of long noncoding RNAs (lncRNAs) and mRNAs in the trabecular meshwork (TM). (A) (B) Heat maps of lncRNA (A) and mRNA (B) microarray expression profiling in TM of normal controls and primary open-angle glaucoma (POAG) patients. (C) (D) Scatter plots of lncRNAs (C) and mRNAs (D) expression profile. (E) (F) Volcano plots of differentially expressed lncRNAs (E) and mRNAs (F) in TM between control group and POAG group. The vertical green lines and horizontal green line indicate cutoff lines for fold change and $\mathrm{P}$ values, respectively (fold change $\leq 0.5$ or $\geq 2$, and $\mathrm{P} \leq 0.05$ )

ENST00000514811, ENST00000552367, ENST00000582505, ENST00000609130, NR_029395, NR_038379, NR_110087, uc002rhy.1, ENST00000586949, and uc. 3 + were significantly upregulated $(* * * \mathrm{P}=0.0002, * \mathrm{P}=0.0191$, $* \mathrm{P}=0.0431, * * * * \mathrm{P}<0.0001, * * * \mathrm{P}=0.0009, * * * * \mathrm{P}<0.0001$,
**** $\mathrm{P}<0.0001, * * * * \mathrm{P}<0.0001, * * * \mathrm{P}=0.0009, * * \mathrm{P}=0.0062$, $* * * \mathrm{P}=0.0004, * * \mathrm{P}=0.006$, respectively) in the POAG group (Fig. 4). Otherwise, there were no significant differences in expression levels of ENST00000521373, ENST00000523317, ENST00000583377, ENST00000585387, NR_003039, NR_024249, NR_027425, and NR_046232 in trabecular meshwork samples between control and POAG groups (Fig. 4). As shown in Fig. 4, most genes (12/20) were consistent with the direction of changes acquired by microarray analysis, confirming the validity of the microarray data.

To further confirm the results, more samples ( 8 cases of normal trabecular meshwork samples and 19 cases of POAG tissue samples) were collected and we confirmed that the expression of ENST00000552367, ENST00000582505, ENST00000609130,NR_029395, NR_038379, and
$\mathbf{A}$

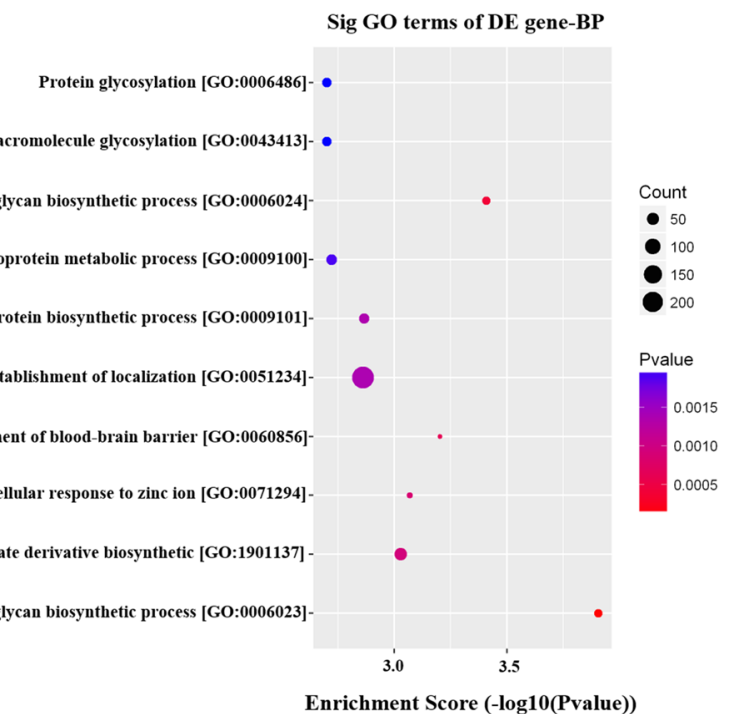

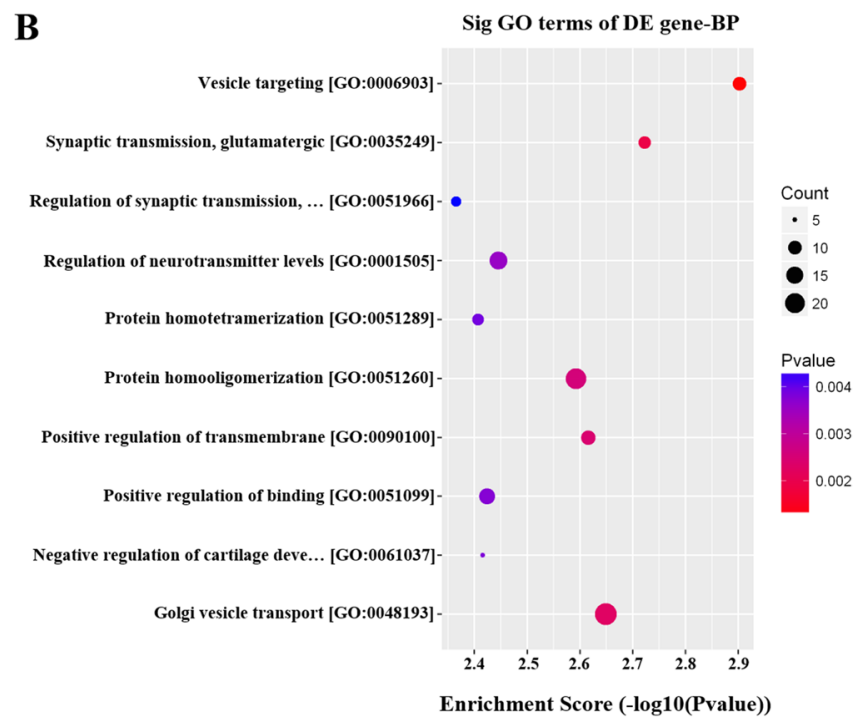

Fig. 2 Gene Ontology (GO) enrichment analyses. (A) Upregulated genes and (B) downregulated genes

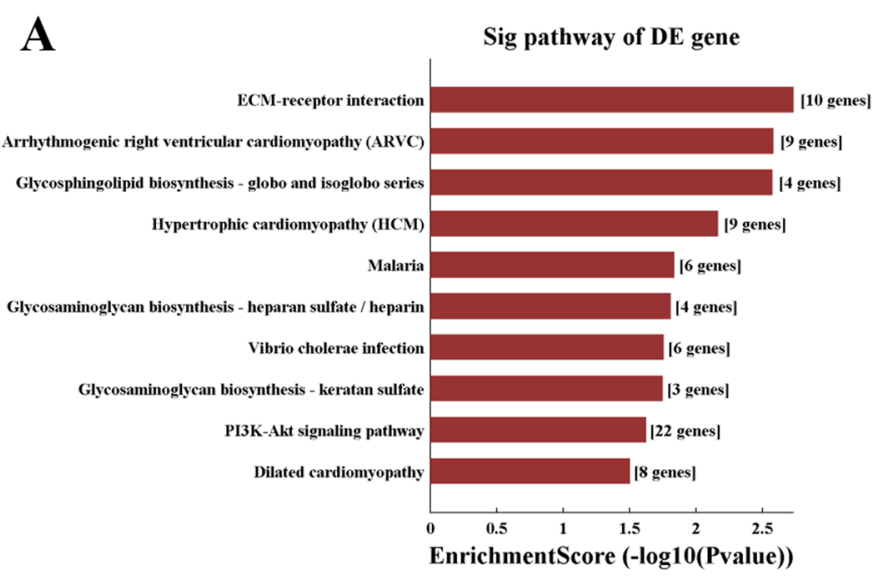

Fig. 3 Kyoto Encyclopedia of Genes and Genomes (KEGG) pathway analyses. (A) KEGG pathway enrichment analyses showing 10 pathways enriched among upregulated genes. (B) KEGG pathway enrichment anal-

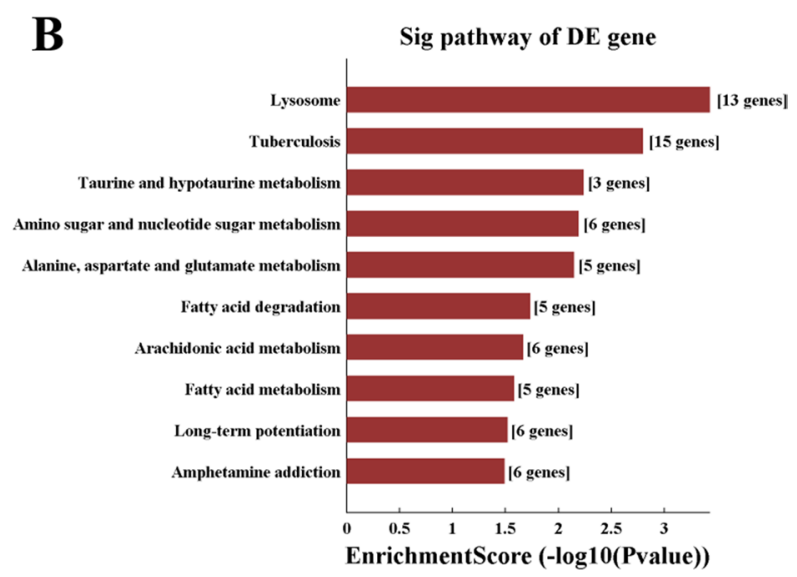

yses showing 10 pathways enriched among downregulated genes. DE, differentially expressed 
Table 2 Primer sequences of 20 lncRNAs

\begin{tabular}{|c|c|}
\hline Primer name & Primer sequence \\
\hline 18S rRNA & $\begin{array}{l}\text { F:5'CAGCCACCCGAGATTGAGCA3' } \\
\text { R:5' TAGTAGCGACGGGCGGTGTG3' }\end{array}$ \\
\hline ENST00000422366 & $\begin{array}{l}\text { F:5' CTCAGGACACCTCCCGTTGC 3' } \\
\text { R:5' TGGGCATCCGTTTGTTTGACT 3' }\end{array}$ \\
\hline ENST00000430429 & $\begin{array}{l}\text { F:5' GCCACAATAGCAGGAAACCTA 3' } \\
\text { R:5' GTCTTGCAGATGGGAGACCA 3' }\end{array}$ \\
\hline ENST00000514811 & $\begin{array}{l}\text { F:5' CTGAAAGGAGCCCCTTGACA 3' } \\
\text { R:5' CGTCTGACCAATGAAAACCGT 3' }\end{array}$ \\
\hline ENST00000521373 & $\begin{array}{l}\text { F:5' GAGTGTGGGGTGGGTCTGAA 3' } \\
\text { R:5' GCACAGGACAGGCGATTTGA 3' }\end{array}$ \\
\hline ENST00000523317 & $\begin{array}{l}\text { F:5' TTGCCGCTGTTGGATGTCA 3' } \\
\text { R:5' CCTGACTTTGCTTTCTCTGACCT 3' }\end{array}$ \\
\hline ENST00000552367 & $\begin{array}{l}\text { F:5' ACCTTACCTTGTCTTGCCCG 3' } \\
\text { R:5' GAGATCACGAGCCGCACTC 3' }\end{array}$ \\
\hline ENST00000582505 & $\begin{array}{l}\text { F:5'ACTGAAGCGACCTTTCCTCG3' } \\
\text { R:5' CGAGGTGCTCCGGGAATC3' }\end{array}$ \\
\hline ENST00000583377 & $\begin{array}{l}\text { F:5' CAGTGGCTCAATCATAGCTCACT 3' } \\
\text { R:5' AGTAACTGGAACCACAGGCACA 3' }\end{array}$ \\
\hline ENST00000585387 & $\begin{array}{l}\text { F:5' CCACCAGACAGAGCAGGATG 3' } \\
\text { R:5' TCTTCCACAAGGGATGGAATG 3' }\end{array}$ \\
\hline ENST00000609130 & $\begin{array}{l}\text { F:5' TTGAGCCTTACGCAGAGGTCT 3' } \\
\text { R:5' TTGGTGGGTAAAGAGGGTGGA 3' }\end{array}$ \\
\hline NR_003039 & $\begin{array}{l}\text { F:5' GCCTCCTTCCACAACTCTCA 3' } \\
\text { R:5' AGGCTGAGTCTCCGAGTGAA 3' }\end{array}$ \\
\hline NR_024249 & $\begin{array}{l}\text { F:5' AGCCCAGAAGCCATCGTGTC 3' } \\
\text { R:5' TGATCCCAGCCCGGCATA 3' }\end{array}$ \\
\hline NR_027425 & $\begin{array}{l}\text { F:5' GTGCCACAACGGGAATCTTG 3' } \\
\text { R:5' ATCAAATTGGTGCCTGGGGTA 3' }\end{array}$ \\
\hline NR_029395 & $\begin{array}{l}\text { F:5' AACAGAGCAACAGCAAGTACAT 3' } \\
\text { R:5' CTGGGAACCTATGAACATTCT 3' }\end{array}$ \\
\hline NR_038379 & $\begin{array}{l}\text { F:5' TACTTTGTGCCAGGGCCTTAT 3' } \\
\text { R:5' TCTTTCCCAACTAAACCGTGAG 3' }\end{array}$ \\
\hline NR_046232 & $\begin{array}{l}\text { F:5' GAGCACTGAGGACCCTTCTTG 3' } \\
\text { R:5' AGCCCACTGACACCTTGACTT 3' }\end{array}$ \\
\hline NR_110087 & $\begin{array}{l}\text { F:5' AGCAGTCCACCCCTGGCTG 3' } \\
\text { R:5' CCAAATAGCTTGCAGTGCTCTGT 3' }\end{array}$ \\
\hline uc002rhy.1 & $\begin{array}{l}\text { F:5' GAAAGTCGGATGCTGAAGATG 3' } \\
\text { R:5'GCAGGTAGAGTAGAGTCTGAGGG 3' }\end{array}$ \\
\hline ENST00000586949 & $\begin{array}{l}\text { F:5' GAAGCAGGAAAAGACAGTCTCTA 3' } \\
\text { R:5' CAGTCTGGTGTACAAGGCAGAA 3' }\end{array}$ \\
\hline uc. $3+$ & $\begin{array}{l}\text { F:5'ATTTGCATAACCCAACCCC3' } \\
\text { R:5' CGATGTCGTCCTAATTCACC3' }\end{array}$ \\
\hline
\end{tabular}

$F$, forward; $R$, reverse

ENST00000586949 was significantly upregulated $(* * * \mathrm{P}=0.0001, * \mathrm{P}=0.0113, * * * * \mathrm{P}<0.0001, * * \mathrm{P}=0.001$, $* * * * \mathrm{P}<0.0001, * * * * \mathrm{P}<0.0001$, respectively) (Fig. 5B, C, D, E, F, H). However, ENST00000422366 and NR_110087 show no significant difference expression levels in trabecular meshwork samples between control and POAG groups (Fig. 5A, G).

\section{Discussion}

Long non-coding RNA demonstrates $\sim$ tenfold lower abundance than mRNAs in a population of cells and characterized as tissue-specific [17]. In addition to higher tissue specificity, lncRNAs are characterized by higher developmental stage specificity [18]. Recent recognition that IncRNAs function in various aspects of cell biology has caused increasing attention on their potential contribution towards diseases etiology [19]. In the glaucoma research area, previous studies have indicated that there is a relationship between IncRNA and glaucoma. Lili Xie et al. [12] identified IncRNAs T267384, ENST00000607393, and T342877 may be potential biomarkers for POAG diagnosis and ENST00000607393 might be a new therapeutic target for trabecular meshwork calcification based on clinical tissues. J. Zhao et al. [11] concluded that IncRNA ANRIL attenuated oxidative injury of human TM cells and activated the mTOR and MEK/ERK pathways, possibly through downregulation of miR-7. Y. Xu et al. [20] found that downregulation of IncRNA GAS5 may maintain retinal ganglion cell survival in glaucoma through the activation of TGF- $\beta$ pathway to promote cell proliferation and differentiation. Haibo Li et al. [21] provided evidence that lncRNA-MALAT1 could inhibit RGC apoptosis in glaucoma through activation of the PI3K/Akt signaling pathway. Shen W. et al. [22] established that oxidative stress-induced lncRNA-RP11-820 plays a key role in regulating the miR3178/MYOD1/ECM axis in HTMCs. Moreover, our previous work [23] had proved that knockdown of lncRNA NR_003923 in human Tenon's capsule fibroblast cells (HTFs) inhibited TGF- $\beta$-induced cell migration, proliferation, fibrosis, and autophagy and overexpression of IL22RA1 enhanced HTF migration and proliferation. Therefore, NR_003923 and IL22RA1 might contribute to glaucoma progression.

However, detailed analyses on expression profiling of lncRNAs in TM of POAG patients have not yet to be reported. Glaucoma has a complex pathogenesis and its symptoms are associated with the long-term intraocular pressure and damage, as well as apoptosis of retinal ganglion cells caused by various pathological factors [24]. Among these multiple factors, IOP, the major risk one for the development and progression of glaucoma, is closely associated with TM tissue [25]. The TM is a series of fenestrated beams and sheets of the extracellular matrix and is responsible for draining the aqueous humor from the eye via the anterior chamber. Therefore, TM tissue plays a crucial role in the development and progression of glaucoma [26]. It would be more convincing to collect TM tissue rather than other ocular tissues for further microarray analyses. This study is the largest comparison of lncRNA expression in the TM of normal controls and POAG patients reported to date. 
Table 3 A collection of lncRNAs detected using microarray in POAG patients
Fig. 4 Real-time quantitative PCR validation of 201 lncRNAs in TM samples from control group and POAG group. Data are expressed as means $\pm \mathrm{SD}$

\begin{tabular}{|c|c|c|c|c|c|c|}
\hline Seqname & Gene Symbol & Type & Source & Chrom & Fold Change & P-value \\
\hline ENST00000422366 & HCG25 & noncoding & GENCODE & chr6 & 6.295 & 0.000 \\
\hline ENST00000430429 & $\begin{array}{l}\text { AC0988 } \\
28.2\end{array}$ & noncoding & GENCODE & chr2 & 8.012 & 0.001 \\
\hline ENST00000514811 & CTB-174D11.2 & noncoding & GENCODE & chr5 & 3.062 & 0.000 \\
\hline ENST00000521373 & СТВ-43E15.2 & noncoding & GENCODE & chr5 & 3.462 & 0.000 \\
\hline ENST00000523317 & RP11-513H8.1 & noncoding & GENCODE & chr8 & 9.207 & 0.000 \\
\hline ENST00000552367 & RP11-290L1.3 & noncoding & GENCODE & $\operatorname{chr} 12$ & 3.587 & 0.002 \\
\hline ENST00000582505 & RP11-180P8.1 & noncoding & GENCODE & $\operatorname{chr} 17$ & 4.507 & 0.007 \\
\hline ENST00000583377 & RP11-848P1.5 & noncoding & GENCODE & chr17 & 8.063 & 0.000 \\
\hline ENST00000585387 & RP11-47L3.1 & noncoding & GENCODE & $\operatorname{chr} 17$ & 3.514 & 0.000 \\
\hline ENST00000609130 & $\begin{array}{l}\text { RP11-127 } \\
5 \mathrm{H} 24.2\end{array}$ & noncoding & GENCODE & chr7 & 3.421 & 0.000 \\
\hline $\begin{array}{l}\text { NR_00 } \\
3039\end{array}$ & $\begin{array}{l}\text { GLY } \\
\text { CAM1 }\end{array}$ & noncoding & RefSeq & $\operatorname{chr} 12$ & 3.403 & 0.000 \\
\hline $\begin{array}{l}\text { NR_02 } \\
4249\end{array}$ & $\begin{array}{l}\text { FAM } \\
86 \mathrm{C} 2 \mathrm{P}\end{array}$ & noncoding & RefSeq & $\operatorname{chr} 11$ & 3.578 & 0.001 \\
\hline $\begin{array}{l}\text { NR_02 } \\
7425\end{array}$ & FAM66D & noncoding & RefSeq & chr8 & 5.053 & 0.000 \\
\hline $\begin{array}{l}\text { NR_02 } \\
9395\end{array}$ & IGLL3P & noncoding & RefSeq & $\operatorname{chr} 22$ & 3.317 & 0.000 \\
\hline $\begin{array}{l}\text { NR_03 } \\
8379\end{array}$ & $\begin{array}{l}\text { LOC } \\
554,206\end{array}$ & noncoding & RefSeq & $\operatorname{chr} 16$ & 4.230 & 0.001 \\
\hline $\begin{array}{l}\text { NR_04 } \\
6232\end{array}$ & $\begin{array}{l}\text { LINC } \\
01,298\end{array}$ & noncoding & RefSeq & chr8 & 3.719 & 0.001 \\
\hline $\begin{array}{l}\text { NR_11 } \\
0087\end{array}$ & $\begin{array}{l}\text { LOC10 } \\
1,927,497\end{array}$ & noncoding & RefSeq & chr7 & 5.512 & 0.000 \\
\hline uc002rhy.1 & $\begin{array}{l}\text { AK125 } \\
769\end{array}$ & noncoding & $\begin{array}{l}\text { UCSC_- }_{-} \\
\text {knowngene }\end{array}$ & chr2 & 6.741 & 0.000 \\
\hline $\begin{array}{l}\text { ENST00000586949 } \\
\text { uc. } 3+\end{array}$ & $\begin{array}{l}\text { RP11-879F14.2 } \\
\text { uc. } 3\end{array}$ & $\begin{array}{l}\text { noncoding } \\
\text { noncoding }\end{array}$ & $\begin{array}{l}\text { GENCODE } \\
\text { UCR }\end{array}$ & $\begin{array}{l}\text { chr18 } \\
\text { chr1 }\end{array}$ & $\begin{array}{l}3.112 \\
5.113\end{array}$ & $\begin{array}{l}0.000 \\
0.000\end{array}$ \\
\hline
\end{tabular}

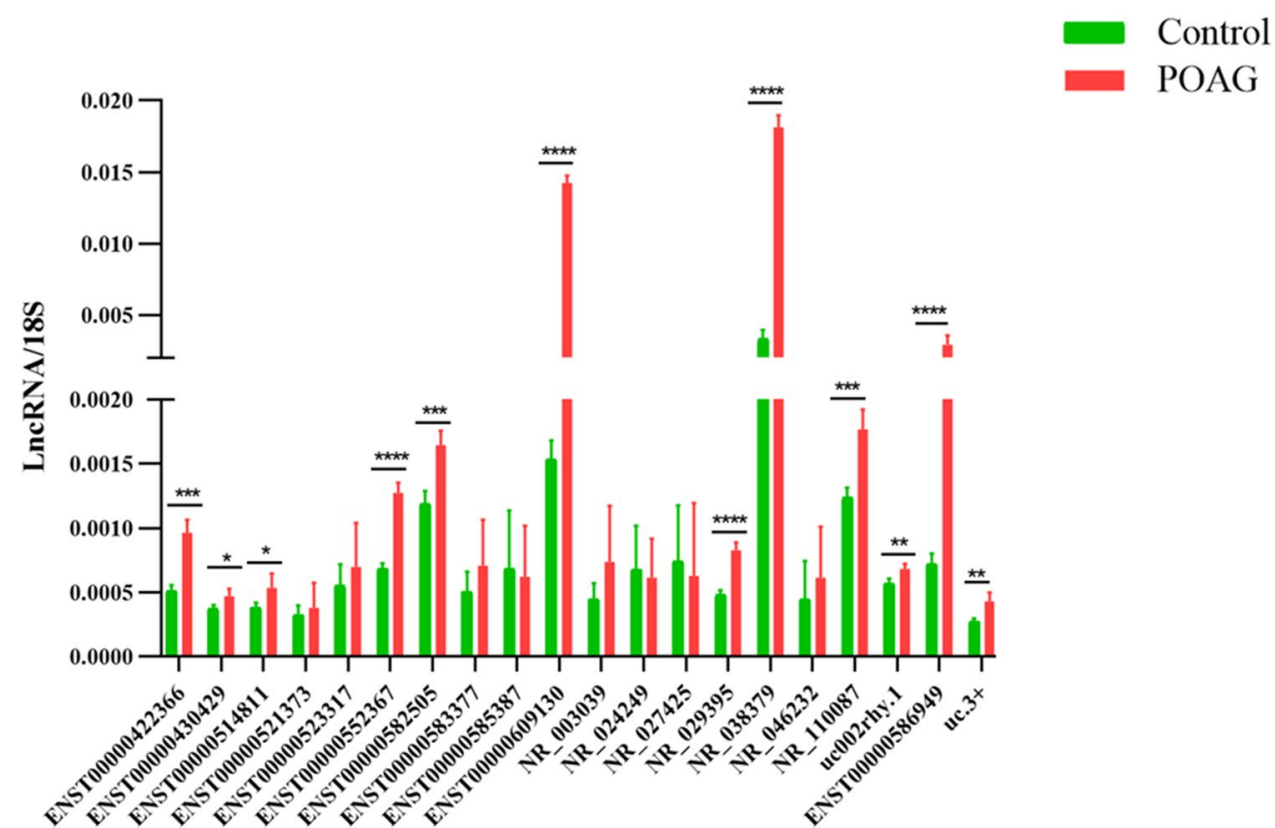



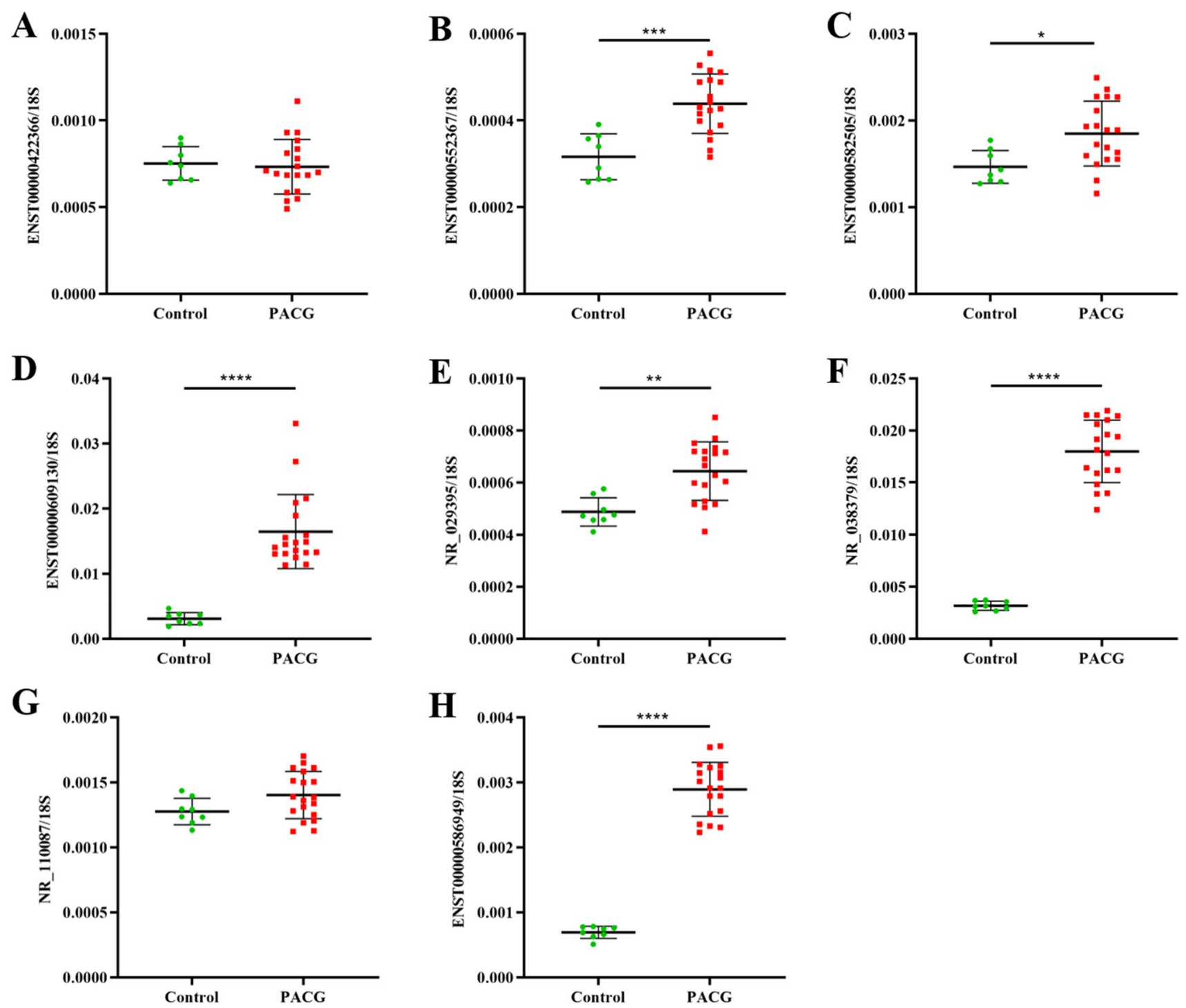

Fig. 5 Real-time quantitative PCR showing expression of ENST00000422366 (A), ENST00000552367 (B), ENST00000582505 (C), ENST00000609130 (D), NR_029395 (E), NR_038379 (F),
NR_110087 (G), and ENST00000586949 (H) in TM of control group and POAG group. Data are expressed as means $\pm \mathrm{SD}$

should be further verified through experiment in vivo and vitro.

\section{Conclusions}

We conclude that IncRNAs ENST00000552367, ENST00000582505, ENST00000609130, NR_029395, NR_038379, and ENST00000586949 may play essential roles in the development of POAG.

Acknowledgements This work was supported in part by the National Natural Science Foundation of China (Grant No. 81970801, Grant No. 81670859 to X.D.), Science and Technology Foundation of Changsha 
Hunan, China (Grant No. kh1801229 to X.D.), and Natural Science Foundation of Hunan Province, China (Grant No. 2019JJ40001 to X.D.).

\section{Declarations}

Ethical approval and consent to participate All procedures performed in studies involving human participants were in accordance with the ethical standards of the Second Xiangya Hospital research committee and with the 1964 Helsinki declaration and its later amendments or comparable ethical standards. Informed consent was obtained from all individual participants included in the study.

Competing interests The authors declare no competing interests.

Open Access This article is licensed under a Creative Commons Attribution 4.0 International License, which permits use, sharing, adaptation, distribution and reproduction in any medium or format, as long as you give appropriate credit to the original author(s) and the source, provide a link to the Creative Commons licence, and indicate if changes were made. The images or other third party material in this article are included in the article's Creative Commons licence, unless indicated otherwise in a credit line to the material. If material is not included in the article's Creative Commons licence and your intended use is not permitted by statutory regulation or exceeds the permitted use, you will need to obtain permission directly from the copyright holder. To view a copy of this licence, visit http://creativeco mmons.org/licenses/by/4.0/.

\section{References}

1. Resnikoff S, Keys TU (2012) Future trends in global blindness. Indian J Ophthalmol 60:387-395

2. Quigley HA (2006) The number of people with glaucoma worldwide in 2010 and 2020. Br J Ophthalmol 90:262-267

3. Jonas JB, Aung T, Bourne RR et al (2017) Glaucoma. Lancet 390:2183-2193

4. Aung T, Khor CC (2016) Glaucoma genetics: recent advances and future directions. Asia Pac J Ophthalmol (Phila) 5:256-259

5. Fan BJ, Leung YF, Wang N et al (2004) Genetic and environmental risk factors for primary open-angle glaucoma. Chin Med J (Engl) 117:706-710

6. Kountouras J, Zavos C, Grigoriadis N et al (2008) Helicobacter pylori infection as an environmental familial clustering risk factor for primary open-angle glaucoma. Clin Exp Ophthalmol 36:296-7 author reply 297

7. Elze T, Baniasadi N, Jin Q et al (2017) Ametropia, retinal anatomy, and OCT abnormality patterns in glaucoma. 1 . Impacts of refractive error and interartery angle. J Biomed Opt 22:1-11

8. Wostyn P, De Groot V, Van Dam D et al (2017) Alzheimer's disease and glaucoma: can glymphatic system dysfunction underlie their comorbidity? Acta Ophthalmol 95:e244-e245

9. Kamat SS, Gregory MS, Pasquale LR (2016) The role of the immune system in glaucoma: bridging the divide between immune mechanisms in experimental glaucoma and the human disease. Semin Ophthalmol 31:147-154
10. Zou Y, Li C, Shu F et al (2015) lncRNA expression signatures in periodontitis revealed by microarray: the potential role of lncRNAs in periodontitis pathogenesis. J Cell Biochem 116:640-647

11. Zhao J, Sun H, Zhang JM et al (2019) Long non-coding RNA ANRIL down-regulates microRNA-7 to protect human trabecular meshwork cells in an experimental model for glaucoma. Eur Rev Med Pharmacol Sci 23:3173-3182

12. Xie L, Mao M, Wang C et al (2019) Potential biomarkers for primary open-angle glaucoma identified by long noncoding RNA profiling in the aqueous humor. Am J Pathol 189:739-752

13. Burdon KP, Crawford A, Casson RJ et al (2012) Glaucoma risk alleles at CDKN2B-AS1 are associated with lower intraocular pressure, normal-tension glaucoma, and advanced glaucoma. Ophthalmology 119:1539-1545

14. Hassan H, Shanak S (2019) GOTrapper: a tool to navigate through branches of gene ontology hierarchy. BMC Bioinformatics 20:20

15. Kanehisa M (2002) The KEGG database. Novartis Found Symp 247:91-101 discussion 101-3, 119-28, 244-52

16. Ihnatova I, Budinska E (2015) ToPASeq: an R package for topology-based pathway analysis of microarray and RNA-Seq data. BMC Bioinformatics 16:350

17. Cabili MN, Trapnell C, Goff L et al (2011) Integrative annotation of human large intergenic noncoding RNAs reveals global properties and specific subclasses. Genes Dev 25:1915-1927

18. Yan L, Yang M, Guo H et al (2013) Single-cell RNA-Seq profiling of human preimplantation embryos and embryonic stem cells. Nat Struct Mol Biol 20:1131-1139

19. Ma L, Cao J, Liu L et al (2019) LncBook: a curated knowledgebase of human long non-coding RNAs. Nucleic Acids Res 47:D128-D134

20. Xu Y, Xing YQ (2018) Long non-coding RNA GAS5 contributed to the development of glaucoma via regulating the TGF-beta signaling pathway. Eur Rev Med Pharmacol Sci 22:896-902

21. Li HB, You QS, Xu LX et al (2017) Long non-coding RNAMALAT1 mediates retinal ganglion cell apoptosis through the $\mathrm{PI} 3 \mathrm{~K} /$ Akt signaling pathway in rats with glaucoma. Cell Physiol Biochem 43:2117-2132

22. Shen W, Huang B, He Y et al (2019) Long non-coding RNA RP11-820 promotes extracellular matrix production via regulating miR-3178/MYOD1 in human trabecular meshwork cells. FEBS J 287(5):978-990. https://doi.org/10.1111/febs.15058

23. Zhao Y, Zhang F, Pan Z et al (2019) LncRNA NR_003923 promotes cell proliferation, migration, fibrosis, and autophagy via the miR-760/miR-215-3p/IL22RA1 axis in human Tenon's capsule fibroblasts. Cell Death Dis 10:594

24. Tham YC, Li X, Wong TY et al (2014) Global prevalence of glaucoma and projections of glaucoma burden through 2040: a systematic review and meta-analysis. Ophthalmology 121:2081-2090

25. Acott TS, Kelley MJ, Keller KE et al (2014) Intraocular pressure homeostasis: maintaining balance in a high-pressure environment. J Ocul Pharmacol Ther 30:94-101

26. Vranka JA, Kelley MJ, Acott TS et al (2015) Extracellular matrix in the trabecular meshwork: intraocular pressure regulation and dysregulation in glaucoma. Exp Eye Res 133:112-125

Publisher's note Springer Nature remains neutral with regard to jurisdictional claims in published maps and institutional affiliations. 


\section{Authors and Affiliations}

\section{Feng Zhang ${ }^{1} \cdot$ Yang Zhao ${ }^{2,3} \cdot$ Mengdan $\mathrm{Cao}^{4} \cdot \mathrm{Xu} \mathrm{Jia}^{5} \cdot$ Zheng Pan $^{4} \cdot$ Dengming Zhou ${ }^{4} \cdot \mathrm{Ke} \mathrm{Liu}^{4} \cdot \mathrm{Xuanchu} \mathrm{Duan}^{2,3}$ (1)}

1 Department of Ophthalmology, The Third Xiangya Hospital, Central South University, Changsha, Hunan Province, China

2 Aier School of Ophthalmology, Central South University, Changsha, Hunan Province, China

3 Changsha Aier Eye Hospital, Changsha, Hunan Province, China
4 Department of Ophthalmology, The Second Xiangya Hospital, Central South University, Changsha, Hunan Province, China

5 Affiliated Hospital of Guizhou Medical University, Guiyang, Guizhou Province, China 